\title{
The Comparison of Stereological Calculations of Tumour Volume with Prognostic Parameters in Breast Cancer
}

\author{
Aslıhan Duman ${ }^{1}$, Cemal Gündoğdu ${ }^{2,}$, Elif Demirci ${ }^{2}$ \\ ${ }^{1}$ Department of Pathology, Medical Faculty of Giresun University, Giresun, Turkey \\ ${ }^{2}$ Department of Pathology, Medical Faculty of Atatürk University, Erzurum, Turkey
}

Email address:

cemgun98@hotmail.com (C. Gündoğdu)

To cite this article:

Aslıhan Duman, Cemal Gündoğdu, Elif Demirci. The Comparison of Stereological Calculations of Tumour Volume with Prognostic Parameters in Breast Cancer. Science Research. Vol. 4, No. 1, 2016, pp. 1-6. doi: 10.11648/j.sr.20160401.11

\begin{abstract}
Purpose: In this study; we aimed to compare tumor volume with other prognostic determinants by making stereologic estimation. Material and Methods: In the mastectomy materials of 44 patients; tumor volume was calculated with the Cavaliery principle and compared with the classically known other pathologic variables. Results: There is a statistically significant correlation between histologic grade and tumor volume $(\mathrm{p}<0.05)$. Conclusion: It is concluded that one of the reliable parameters in the prognosis of breast cancer may be the estimation of tumor volume.
\end{abstract}

Keywords: Breast, Tumor Burden, Tumor Volume

\section{Introduction}

Breast carcinomas are the most commonly encountered tumours in women and rank first among the causes of death from cancer. The aetiology of breast cancer is known to be multifactorial and genetic predisposition, age and reproductive and hormonal effects are reported as major factors $(1,2)$. The histopathological typing and grading of breast carcinomas is performed in accordance with the 2003 tumour classification of the World Health Organisation (WHO) (3).

In current practice, several prognostic parameters are evaluated, including the histopathological type of the tumour. Prognostic parameters include tumour diameter, metastatic lymph nodes, lymphovascular (LV) invasion, histological grade (HG), nuclear grade, oestrogen and progesterone receptor levels, c-erbB2 immunoreactivity, perinodal invasion, and peritumoural lymphocytic response $(4,5)$. Among these, macroscopic diameter of tumours is regarded as an important prognostic criterion. Determination of the macroscopic tumour dimension is closest to the real dimension of prognostic importance. In the present study, stereology was used for the estimation of tumour volume (TV) (4).

Stereology is a method that provides information on the geometric and numeral features of three-dimensional viable and non-viable structures by utilizing their appearances on a two-dimensional platform $(6-8,20)$. The Cavalieri principle, which is a stereological volume estimation method, enables the determination of the volume of objects with imperfect shape $(9,10)$.

The present study was aimed at the stereological estimation of TV with the aid of prognostic parameters in breast tumours. Mastectomy materials of 44 patients constituted the material of the study. No correlation was determined between LV invasion, lymph node involvement and TV. On the other hand, it was ascertained that HG and TV were significantly correlated with each other.

In conclusion, it is considered that the measurement of TV is of prognostic value in breast tumours.

\section{Materials and Methods}

The present study was conducted on 44 cases diagnosed with breast cancer between the years 2007 - 2009, which were treated with modified radical mastectomy and dissection of the axillary lymph node (ALN). The mastectomy materials of the patients included in the study were fixed in $10 \%$ buffered formaldehyde for 24 hours (Figure 1), and the Cavalieri principle was applied for the estimation of TV. When applying the Cavalieri method, on the rear surface, each mastectomy material was cut into equal and parallel $1 \mathrm{~cm}$ thick sections (Figure 1). These sections were placed side-by-side without altering the 
sequencing for the superimposition of the point grid (Figure 2). A common point counting grid with $1 \mathrm{~cm}$ distance between test points was used for the estimation of the volume of the tumour. The grid system was superimposed, covering the sectioned surface area, and the circled points were counted (Figure 3).

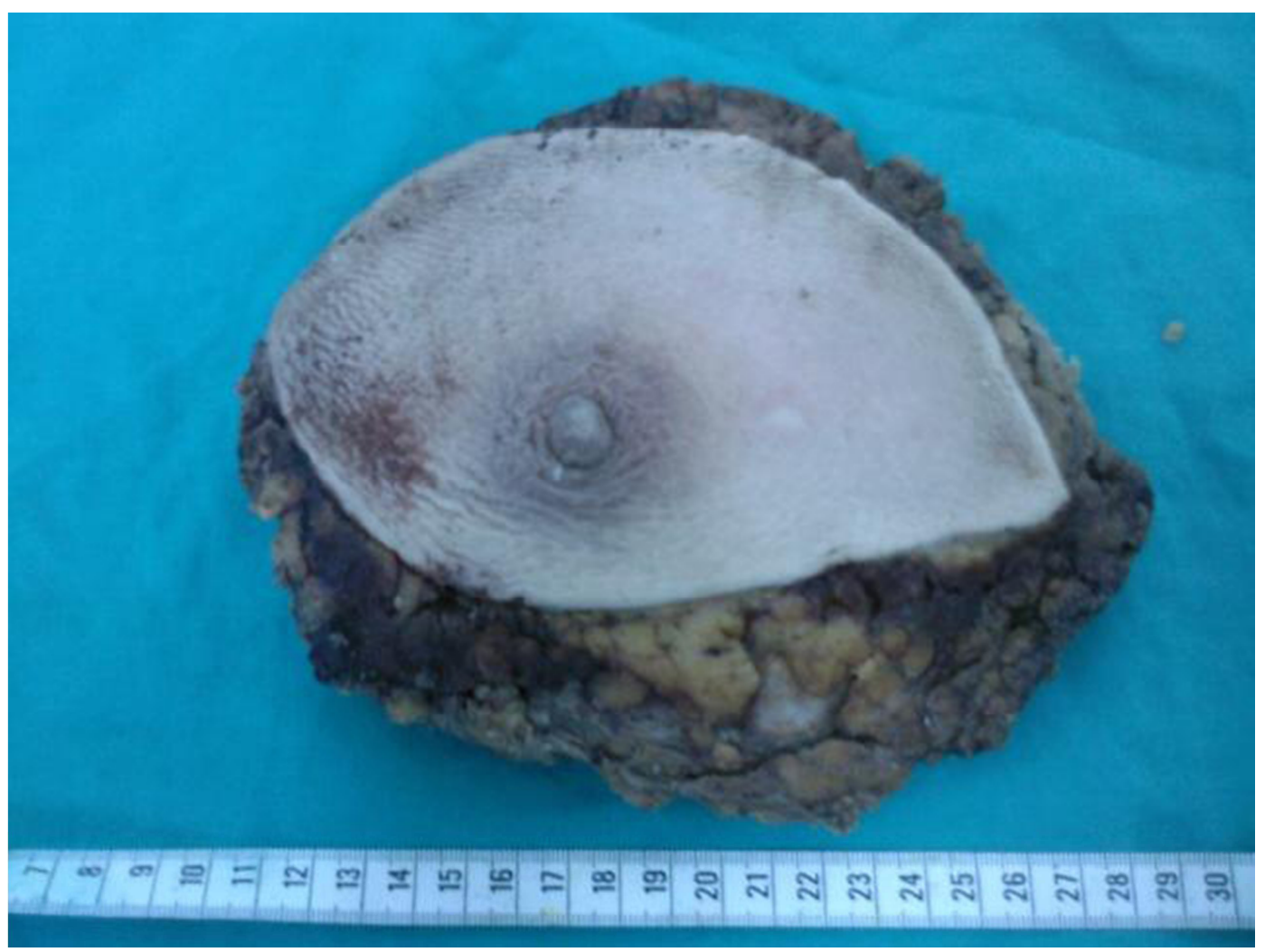

Figure 1. Mastectomy material fixed in 10\% buffered formaldehyde for 24 hours.

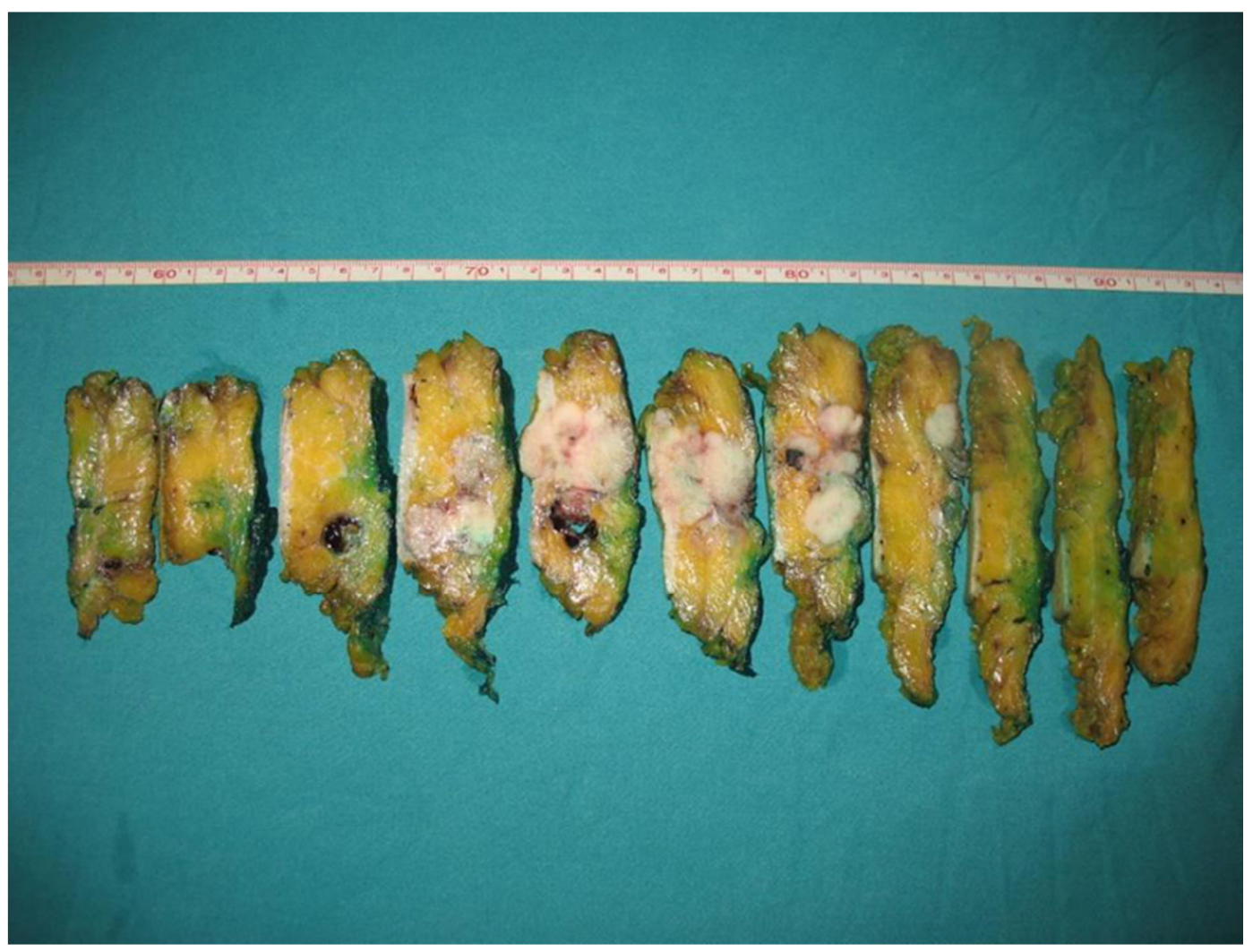

Figure 2. 1 - cm - thickparallel mastectomy sections placed such that the same surface faces upward. 


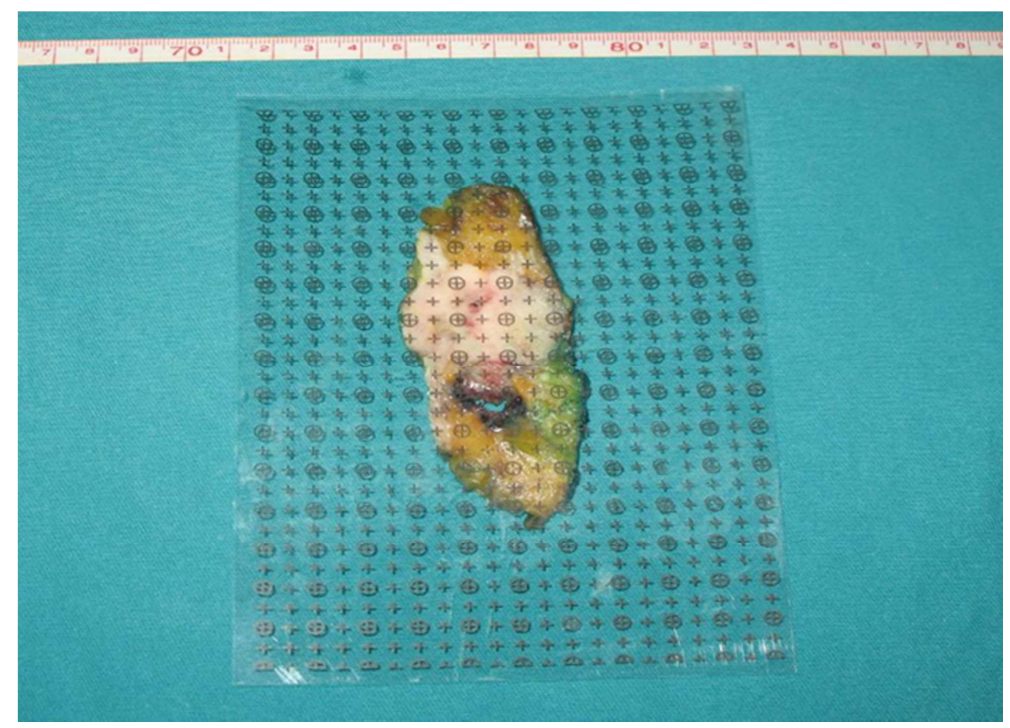

Figure 3. Superimposition of a transparent point grid for the counting of the circled points hitting the section.

The total volume was calculated by placing the total number of points and other measurement criteria in a formula frequently used in Cavalieri estimations. The mathematical equation used is given below:

$$
\mathrm{V}=\mathrm{t} \times \mathrm{a} / \mathrm{p} \times \mathrm{P}
$$

$\mathrm{V}$ : Volume of the section of interest

P: Number of points counted in the cross-sectional area

$\mathrm{t}$ : Mean section thickness $(\mathrm{cm})$

$\mathrm{a} / \mathrm{p}$ : The actual area represented by each point.

In the present study, the number of points counted in the cross-sectional area was placed together with the $t$ (mean section thickness) and $\mathrm{a} / \mathrm{p}$ (actual area represented by each point) parameters in the above formula to calculate the TV for each case.

To exemplify, for a neoplastic area with a $\mathrm{P}$ value of 3 the volume was calculated as follows:

$$
\mathrm{V}=1 \times 1 \times 3 \ldots \mathrm{V}=3 \mathrm{~cm}^{3}
$$

In cases where the neoplastic area involved more than one section, the number of circled points hitting each section was counted and the counts of each section were added to obtain a total number of points. The sum was used in the formula given below for the calculation of the TV.

$$
\mathrm{V}=\mathrm{t} \times \mathrm{a} / \mathrm{p} \times(\mathrm{P} 1+\mathrm{P} 2+\mathrm{P} 3 \ldots \mathrm{Pn})
$$

To exemplify, for neoplastic areas with P1, P2 and P3 values of 2,5 , and 3 , respectively, the tumour volume was calculated as shown below:

$$
\mathrm{V}=1 \times 1 \times(2+5+3) \ldots \mathrm{V}=10 \mathrm{~cm}^{3}
$$

Following the macroscopic measurement of TV, macroscopic sampling was performed for microscopic examination. The preparations prepared by routine processing, embedding, cutting of sections and staining with Hematoxylin-Eosin (H\&E) were assessed by two different pathologists for classical prognostic parameters.

\section{Results}

Of the cases investigated in the present study, $42(95.5 \%)$ were female and $2(4.5 \%)$ were male. Of the mastectomy materials, $19(43.2 \%)$ belonged to the right breast and 25 $(56.8 \%)$ to the left breast. The age distribution of the cases ranged from 22 to 84 , and the mean age of the 44 cases was $51.66 \pm 13.42$. The location of the macroscopic masses in the mastectomy material of the investigated cases was the upper outer quadrant in 15 cases $(34.1 \%)$, the mid-quadrant in 13 cases $(29.5 \%)$, the lower outer quadrant in 6 cases $(13.6 \%)$, the lower inner quadrant in 4 cases $(9.1 \%)$, all quadrants in another 4 cases $(9.1 \%)$ and the upper inner quadrant in 2 cases $(4.5 \%)$. In 3 of the cases $(6.8 \%)$ the tumour was multifocal and in 1 case $(2.3 \%)$ the tumour displayed multicentric localisation.

Histopathological examination of the mastectomy materials pertaining to the 44 cases revealed that 35 of the cases $(79.5 \%)$ were invasive ductal carcinoma (IDC), 7 $(15.9 \%)$ were mixed tumours (MT), and $2(4.5 \%)$ were inflammatory carcinoma (IC). Based on the use of the Nottingham modification of the Bloom-Richardson system, the HG was 2 in 36 of the cases $(81.8 \%$ ) and was 3 in 8 of the cases $(18.2 \%)$. Furthermore, 29 of the cases $(65.9 \%)$ were evaluated negative for comedonecrosis, whilst 15 (34.1\%) were evaluated positive. LV invasion was present in 30 cases (68.2\%), but did not exist in 14 cases $(31.8 \%)$.

The TNM grading of the axillary lymph nodes demonstrated that in 16 of the cases $(36.4 \%)$ there was no metastasis, whilst in $11(25 \%)$ cases the cancer had metastasized to 1- 3 lymph nodes, with metastasis to 4- 9 lymph nodes in another 11 cases $(25 \%)$ and more than 10 lymph nodes in further 6 cases $(13.6 \%)$.

Macroscopic Findings:

Macroscopic masses belonging to the cases were subjected to volume estimation using the Cavalieri method for the determination of the largest diameter. Measurements of all 
cases showed that the largest, smallest and mean diameters were $4.1 \pm 2$, and $1309 \mathrm{~cm}$, respectively; whilst the largest, smallest and mean volumes were $53 \mathrm{~cm}^{3}, 1 \mathrm{~cm}^{3}$ and $12.73 \pm$ $12.633 \mathrm{~cm}^{3}$, respectively.

Correlation of the Size of the Tumour with other Prognostic Parameters:

As regards the correlation between tumour localisation and $\mathrm{TV}$, it was observed in the 44 cases that the tumours located in the upper-outer quadrant, mid-quadrant and all four quadrants were macroscopically larger in volume. The correlation between these two parameters was statistically significant $(\mathrm{P}<0.001)$. However, no statistically significant correlation existed between the largest tumour diameter and the location of the tumour $(\mathrm{P}=0.340)$.

The assessment of the correlation between the histopathological type of the tumour and the location of the tumour in the right- left breast showed that $92 \%$ of the IDC cases were located in the left breast, which was of statistical significance $(\mathrm{P}=0.49)$.

Assessment of the correlation between the total TV and HG demonstrated that the TV of cases with a HG of 3 was larger. This correlation was found to be statistically significant $(\mathrm{P}=0.029)$. However, the largest tumour diameter was not significantly correlated with $\mathrm{HG}(\mathrm{P}=0.188)$.

Assessment for any correlation between LV invasion and TV demonstrated that the 30 cases positive for $\mathrm{LV}$ invasion displayed a mean volume range of $14.90 \pm 13.361 \mathrm{~cm}^{3}$, whilst the 14 cases negative for LV invasion displayed a mean volume range of $8.07 \pm 9.770 \mathrm{~cm}^{3}$. However, although the tumour volumes of the cases positive for LV invasion were greater and this was statistically insignificant $(\mathrm{P}=$ 0.181).

Assessment for any correlation between TV and comedonecrosis showed that the mean TV of the 15 cases positive for comedonecrosis ranged between $16.47 \pm 14.793$ $\mathrm{cm}^{3}$ whilst the tumour volume of the 29 cases negative for comedonecrosis ranged between $10.79 \pm 11.150 \mathrm{~cm}^{3}$. Based on these data, it was considered that TV could be correlated with positiveness for comedonecrosis. However, no statistically significant correlation was determined between comedonecrosis and TV $(\mathrm{P}=0.161)$.

Furthermore, any correlation between TV and metastasis to the axillary lymph nodes was investigated. Accordingly, it was ascertained that TV ranged between $12.56 \pm 13.711 \mathrm{~cm}^{3}$ in 16 cases with no metastasis to the lymph nodes, $11.27 \pm$ $6.214 \mathrm{~cm}^{3}$ in 11 cases with metastasis to $1-3$ lymph nodes, $11.91 \pm 15.865 \mathrm{~cm}^{3}$ in 11 cases with metastasis to $4-9$ lymph nodes, $17.33 \pm 14.010 \mathrm{~cm}^{3}$ in 6 cases with metastasis to 10 and more lymph nodes. The assessment of all 44 cases for tumour metastasis revealed that the mean TV ranged between $12.73 \pm 12.633 \mathrm{~cm}^{3}$. Although it was ascertained that the mean TV of the cases with metastasis to 10 and more lymph nodes ranged between $17.33 \pm 14.010 \mathrm{~cm}^{3}$, no statistically significant correlation existed between TV and lymph node metastasis $(\mathrm{P}=0.590)$.

Investigation of any possible correlation between largest tumour diameter, considered as one of the most significant prognostic parameters, and TV revealed that TV increased with larger tumour diameter. It was determined that largest tumour diameter and TV were significantly correlated with each other $(\mathrm{P}=0.000)$.

In the present study, it was determined that TV and largest tumour diameter were significantly correlated with each other $(\mathrm{p}=0.001)$. It was observed that, as the diameter of the tumour increased so did the volume of the tumour, and with statistical significance. Furthermore, TV and HG were also significantly correlated with each other $(p=0.029)$. It was observed that, as the volume of the tumour increased, the HG also increased significantly. Statistical analyses demonstrated that tumour location and TV were significantly correlated with each other $(p<0.001)$.

Based on all these data, it is considered that stereological volume estimation using the Cavalieri principle is a simple, practical and readily applicable method, which is more reliable than largest tumour diameter in assessing the prognosis of breast tumours.

\section{Discussion}

Major prognostic factors of breast cancer, which are based on multiple clinical and pathological features, and have effect on both treatment results and the biological behaviour of the patient, include tumour diameter, histopathological type of tumour, presence of ALN metastasis and number of metastases, $\mathrm{HG}$, presence of oestrogen and progesterone receptor proteins, and c-erb B2 immunoreactivity $(2,4)$. According to the latest breast cancer classification of the WHO published in 2003, the most frequently observed type of breast cancer is IDC (75\%) (3), and it has been reported that this type of cancer is observed slightly greater in the left breast compared to the right breast (approximately $1.07 / 1$ ) with $40-50 \%$ of the cases located in the upper outer quadrant (2).

In compliance with previous literature reports, in the present study, it was determined that of the 44 cases, 35 $(79.5 \%)$ were IDC, 7 (15.9\%) were MT and 2 (4.5\%) were IC. Furthermore, it was ascertained that the tumours were determined in the mastectomy material of the left breast in 25 cases $(56.8 \%)$ and the mastectomy material of the right breast in 19 cases $(43.2 \%)$. The localisation site of the macroscopic masses was the upper outer quadrant in 15 cases (34.1\%), the mid-quadrant in 13 cases $(29.5 \%)$, the lower outer quadrant in 6 cases $(13.6 \%)$, the lower inner quadrant in 4 cases $(9.1 \%)$, all quadrants in 4 cases $(9.1 \%)$, and the upper inner quadrant in 2 cases $(4.5 \%)$. Statistical assessment showed that, TV and tumour localisation were significantly correlated with each other $(\mathrm{P}<0.001)$.

In a study conducted by Andea et al. (11) on breast cancer specimens with 122 multiple macroscopic nodules, based on the comparison of the status of the axillary lymph nodes by volume and area calculations, it was determined that tumours with a single focus and those with multiple macroscopic nodules, which were ascertained to have similar volume and 
area, involved a greater number of lymph node metastases. In this respect, the researchers indicated that, even if they have a small volume, breast tumours with multiple macroscopic nodules could display different biological behaviour.

Among the cases investigated in the present study, there were 4 cases with multiple macroscopic nodules, 1 with multicentric and 3 with multifocal localisation. No statistically significant correlation was determined to exist between total volume measurements and ALN metastasis ( $P$ $=0.692$ ).

Ahmad et al. (12), in a study conducted on 120 mastectomy materials dissected together with their axillary lymph nodes, aimed to score breast carcinomas based on HG, measurement of tumour size, determination of the status of ALN, staging and Nottingham prognostic index scoring. Accordingly, of the 120 cases, 5, 91, and 24 were determined to be of grades 1, 2, and 3 respectively. It was ascertained that, most of the cases of a higher HG were T2 or T3 and displayed an increased number of metastatic lymph nodes.

$\mathrm{HG}$ has been investigated in various studies as a prognostic factor of breast carcinomas. In mastectomized patients with higher $\mathrm{HG}$, metastasis to 4 or more lymph nodes, systemic recurrences and death from metastatic disease were observed more frequently in comparison to patients with lower HG $(13,14)$.

In the present study, TV and HG were determined to be significantly correlated with each other $(\mathrm{P}=0.029)$. When the metastatic lymph nodes of the 44 cases histologically graded as 2 and 3 were compared, it was observed that no statistically significant correlation existed $(\mathrm{P}=0.537)$. Likewise, no significant correlation existed between largest tumour diameter and $\mathrm{HG}(\mathrm{P}=0.188)$.

In another study, Westenend et al. grouped 100 cases as those with and without distant metastasis such that each of the two groups comprised of 50 patients, and found out that vascular invasion and tumour diameter were both closely related to metastatic potential and prognosis (15).

In a study conducted by Tezuka et al. on 132 cases with breast tumour, the lymphovascular epithelium was immunohistochemically stained with the D2-40 antibody, and vascular invasion was assessed after the application of staining with H\&E and D2-40. Based on the results obtained with both staining methods, the researchers reported that LV invasion, lymph node metastasis and local recurrence were all closely related to each other (16).

In the present study, the mean volume measurements of the 30 cases positive for LV invasion ranged between $14.90 \pm$ $13.361 \mathrm{~cm}^{3}$, whilst the same measurements ranged between $8.07 \pm 9.770 \mathrm{~cm}^{3}$ in the 14 cases negative for LV invasion. However, although tumour volumes were larger in cases positive for LV invasion, this was statistically insignificant ( $\mathrm{P}$ $=0.181)$. Furthermore, LV invasion and lymph node metastasis were determined not to be significantly correlated with each other $(\mathrm{P}=0.338)$.

Lee et al. reported that, of tumours with a diameter smaller than $10 \mathrm{~mm}$, only $3 \%$ presented with local recurrence and only $5 \%$ presented with distant metastasis (17).
In a similar study conducted by Silverstein et al., it was determined that tumours with smaller diameter displayed metastasis at a rate of $3 \%$, whilst in tumours with a diameter greater than $51 \mathrm{~mm}$, the rate of lymph node metastasis increased by $60 \%$ (18).

The assessment of any possible correlation between mean volume measurements and lymph node metastasis revealed that, although TV was larger in cases with metastases to 10 and more lymph nodes, no statistically significant correlation existed between TV and lymph node metastasis $(\mathrm{P}=0.590)$.

Akbaş et al. measured the volumes of axial and coronal MR sections and 9 breast prostheses using the Cavalieri principle. Based on the results they obtained, the researchers reported that the volume of breast prostheses could be measured without altering routine procedures and by making use of this rapid, simple, accurate and practical technique (10).

Altunkaynak et al. calculated by two different methods with the brain volumes of 10 Sprague Dawley rats. First, all brain slices scanned with a scanner and their images shown on PC monitor and brain volume calculated on these images. Second, all brain slices photographed in 10x magnification with a microscope camera, and brain volumes estimated based on these micrographs. There was no statistically difference between to techniques $(\mathrm{P}>0.05)$. This study demonstrates that personel computer scaning of serial tissue sections allows for easy and reliable volume determination based on Cavalieri method (19).

In the present study, after the mastectomy materials entered the macroscopy laboratory, no alteration was made in routine procedures. Each material was cut into $1 \mathrm{~cm}-$ thick slices with an aim of using a common point counting grid to estimate the TV. The grid system was superimposed, covering the sectioned surface area, and the circled points were counted. As the distance between the test points in the counting grid was $1 \mathrm{~cm}$ and the sections were of a thickness of $1 \mathrm{~cm}$, TV calculations could be made practically within a short period of time, using the relevant formula.

Earlier, results were given based on the measurement of the largest tumour diameter as a prognostic parameter. However, repetitive measurements have shown that the same value is not produced. In stereology, the same value is produced in repetitive measurements only if the error coefficient is calculated.

Furthermore, although largest tumour diameter may be of value in spheroidal lesions, it proves to be insufficient in linearly growing lesions. However, TV is considered as a realistic parameter representative of the neoplastic mass.

In the present study, it was considered that, in breast tumours, macroscopic volume could be used as an indicative parameter for prognosis and lifetime expectancy.

\section{References}

[1] Tokatlı Z. F, Türe M, Ömürlü K, Alas R. Ç, Uzal M. C. developing and comparing two different prognostic indexes for predicting disease-free survival of metastatic breast cancer patients. Turk J Med Sci 2011; 41(5): 769-80. 
[2] Rosai j: Breast in: Rosai and Ackerman's Surgical Pathology. Ninth Edition London: Elsevier Inc. 2004. 1763-1839.

[3] Tavassoli F. A., Devilee P. World Health Organization Classification of Tumours, Pathology and Genetics of Tumours of the Breast and Female Genital Organs. Lyon: IARC Press, 2003.

[4] Rosen PP: Rosen's Breast Pathology, Chapter 1, 12, 14, 34, Lippincott Williams and Wilkins, 2001 second ed.: 1-22, 253256, 325-364, 627-652.

[5] Stierer M, Rosen H, Weber R, Hanak H, Spona J, Tuchlert. Immunohistochemical and Biochemical Measurement of Estrogen and Progesterone Receptors in Primary Breast Cancer Correlation of Histopathology and Prognostic Factors, Annals of Surgery 1993 July; 218: 13-21.

[6] Howard CV, Reed MG. Unbiased Stereology: Three dimensional measurement in microscopy. Oxford, Bios Scientific Publishers. 1998; 39-64.

[7] Gundersen HJG. Stereology of arbitrary particles. A review of unbiased number and size estimators and the presentation of some new ones in memory of William R Thomson. J Microsc. 1986; 143: 3-45.

[8] Odacı E, Yıldırım Ş, Bahadır A, Canan S, Baş O, Bilgiç S, Kaplan S. Yeni Stereolojik Yöntemlerin Olası Hata Kaynakları Ve Çözüm Yolları. Turkiye Klinikleri J Med Sci 2004; 24(1): 78-87.

[9] Canan S, Şahin B, Odacı E, Ünal B, Aslan H, Bilgiç S et al. Toplam Hacim, Hacim Yoğunluğu ve Hacim Oranlarının Hesaplanmasında Kullanılan Bir StereolojikYöntem: Cavalieri Prensibi. Türkiye Klinikleri Tıp bilimleri Dergisi Özel Sayısı; Stereolojide Temel Kavram ve Yöntemler 2002a; 22: 7-14.

[10] Akbas H, Sahin B, Eroglu L, Odaci E, Bilgic S, Kaplan S et al. Estimation of Breast Prosthesis Volume by the Cavalieri Principle Using Magnetic Resonance Images. Aesthetic Plastic Surgery, 2004 September-October; 28: 275-80.
[11] Andea AA, Bouwman D, Wallis T, Visscher DW. Correlation of tumour volume and surface area with lymph node status in patients with multifocal / multicentric breast carcinoma. 2004 Jan 1; 100: 20-7.

[12] Ahmad Z, Khurshid A, Qureshi A, Idress R, Asghar N, Kayani N. Breast carcinoma grading, estimation of tumour size, axillary lymph node status, staging, and Nottingham prognostic index scoring on mastectomy specimens. Indian Journal of Pathology and Microbiology 2009; 52-4: 477-481.

[13] Henson DE. Histological grading of breast cancer: Significance of grade on recurrence and mortality. Arch Pathol Lab Med 1988; 112: 1091-6.

[14] Hopton DS, Thorogood J, Clayden AD. Histological grading of breast cancer: significance of grade on recurrence and mortality. Eur J Surg Oncol 1989; 15: 25-31.

[15] Westenend PJ, Meurs CJC, Damhuis RAM. Tumour size and vascular invasion predict distance metastasis in stage 1 breast cancer grade distinguishes early and late metastasis. Journal of Clinical Pathology. 2005; 58: 196-201.

[16] Tezuka K, Onoda N, Takashima T, Takagaki K, Ishikawa T, Wakasa $\mathrm{T}$ et al: Prognostic significance of lymphovascular invasion diagnosed by lymphatic endothelium immunostaining in breast cancer patients. Oncol Rep. 2007; 17: 997-1003.

[17] Lee AK, Loda M, Mackarem G, Bosari S, Delellis RA, Heatley GJ et al: Lymph node negative invasive breast carcinoma or less in size (T1a, b N0 M0) clinicopathologic features and outcome. Cancer. 1997; 79: 761-771.

[18] Siverstein MJ, Gierson ED, Waisman JR, Senofsky GM, Colburn WJ, Gamagami P. Axillary lymph node dissection for T1a breast carcinoma: is it indicated? Cancer 1994; 73: 664667.

[19] Altunkaynak ZB, Altunkaynak E, Unal D, Unal B. A novel application for the Cavalieri Principle: A stereological and methodological study. EAJM August 2009; 41. 